\title{
Isolation and Characterization of Full-Length Phenylalanine Ammonium Lyase and Cinnamyl Alcohol Dehydrogenase Genes Involved in Lignin Biosynthesis of Erianthus Arundinaceus ${ }^{+}$
}

\author{
Lakshmi Kasirajan ${ }^{1}$, Prathima Perumal Thirugnanasambandam 1,*, Agnelo Furtado ${ }^{2}$, \\ Frikkie C. Botha ${ }^{3}$ and Robert J. Henry ${ }^{2}$ \\ 1 ICAR Sugarcane Breeding Institute, Coimbatore 641007, India; lakshmimbb@gmail.com (L.K.) \\ 2 Queensland Alliance for Agriculture and Food Innovation, The University of Queensland, Brisbane, \\ QLD 4072, Australia; a.furtado@uq.edu.au (A.F.); robert.henry@uq.edu.au (R.J.H.) \\ 3 Sugar Research Australia, Indooroopilly 4068, Australia; fbotha@sugarreserachcom.au \\ * Correspondence: prathimasambandam@gmail.com \\ † Presented at the Third International Tropical Agriculture Conference (TROPAG 2019), Brisbane, Australia, \\ 11-13 November 2019.
}

Published: 7 April 2020

\begin{abstract}
Lignocellulosic biomasses available in abundance is the most promising raw material for alternate energy production considering the issues of dwindling oil prices, and global warming. Recently, Erianthus arundinaceous has been identified as a potential target for second generation biofuel crop due to its high biomass production, and adaptability to extreme growth environments. Lignin is a major plant cell wall polymer indispensable for plant growth and development, however it hinders the saccharification of lignocellulosic biomass. Based on the previous transcriptome studies in a set of sugarcane genotypes differing for lignin content, genes encoding cinnamyl alcohol dehydrogenase (CAD), and Phenylalanine ammonia lyase (PAL) genes playing major roles in genetic regulation of lignin production have been cloned and characterized from an Erianthus clone IK 76-81. The genomic region of EriCAD was $3524 \mathrm{bp}$ sequence containing four exons and three introns, among which the exon $1 \& 2$ of 88 and 80 bp were conserved with sorghum and Miscanthus CADs. The coding region of CAD was identified with $1086 \mathrm{bp}$ open reading frame (ORF), a $68 \mathrm{bp} \mathrm{5}$ untranslated region (UTR), and a 86 bp 3' untranslated region (UTR). In the PROSITE analysis, a zinc-containing alcohol dehydrogenase signature (GHEVVGEVVEVGPEV) and an NADP-binding domain motif (GLGGLG) was identified. Similarly sequence analysis of PAL showed an ORF of $2106 \mathrm{bp}$ encoding for 702 amino acid residues. It was flanked by $172 \mathrm{bp}$ of 5' UTR and $121 \mathrm{bp}$ of 3' UTR. This sequence information on PAL and CAD from Erianthus might be useful for subsequent research on lignin modification for improved biomass conversion.
\end{abstract}

Keywords: lignocellulosic biomass; Erianthus; lignin modification

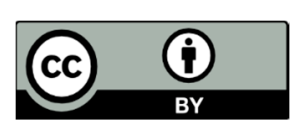

(C) 2020 by the authors. Licensee MDPI, Basel, Switzerland. This article is an open access article distributed under the terms and conditions of the Creative Commons Attribution (CC BY) license (http://creativecommons.org/licenses/by/4.0/). 